\title{
Alkaline Phosphatase to Creatinine Ratio Measurement
}

National Cancer Institute

\section{Source}

National Cancer Institute. Alkaline Phosphatase to Creatinine Ratio Measurement. NCI

Thesaurus. Code C79438.

The determination of the ratio of alkaline phosphatase compared to creatinine present in a sample. The measurement may be expressed as a ratio or percentage. 